\title{
Stabilization of Tollmien-Schlichting Waves by Mode Interaction
}

\author{
Carlos Martel, Eusebio Valero, and José M. Vega \\ E.T.S.I. Aeronáuticos, Universidad Politécnica de Madrid, Plaza Cardenal \\ Cisneros, 3. 28040-Madrid, Spain \\ vega@fmetsia.upm.es
}

\section{Introduction}

Decreasing skin friction in boundary layers attached to aircraft wings can have an impact in both fuel consumption and pollutant production, which are becoming crucial to reduce operation costs and meet environmental regulations, respectively. Skin friction in turbulent boundary layers is about ten times that of laminar boundary layers. Thus, an obvious method to reduce friction drag is to delay transition to turbulence, which is a fairly involved process in real aircraft wings [J98]. Transition sis promoted either by Tollmien-Schlichting (TS) and Klebanov (K) modes [K94], with the former playing an essential role. Various methods (e.g., suction [SG00,ZLB04], wave cancellation [WAA01,LG06]) have been proposed to reduce TS modes in laminar boundary layers. Mode interaction methods have been successfully used in fluid systems to control related instabilities, such as the Rayleigh-Taylor instability [LMV01]. Here, we present some recent results on using these methods to control TS modes in a compressible, $2 \mathrm{D}$ boundary layer over a flat plate at zero incidence. A given unstable TS mode can be stabilized by coupling its spatial evolution with that of a second selected stable TS mode, in such a way that the stable mode takes energy from the unstable one and gives a stable coupled evolution of both modes. The coupling device is a wavetrain in the boundary layer, with appropriate wavenumber and frequency, which can be created by an array of oscillators on the wall, and promotes both (i) parametric coupling between the stable and unstable TS modes and (ii) a mean flow that is also stabilizing. Three differences with wave cancelation methods are relevant. Namely, (a) nonlinear terms play an essential role in the process; (b) the unstable TS mode is stabilized (its growth rate is decreased), not just canceled; and (c) stabilization does not depend on the phase of the incoming wave, which implies that active control is not necessary.

This paper is devoted to analyzing the effect and is organized as follows. After formulating the problem in Sect. 2, the stabilizing process is described in 
Sect. 3, where the relevant mode interactions are described. A short description of the numerical tool used to calculate the non stationary flow in a boundary layer attached to a flat plate, and the post processing tool developed to filter the amplitudes of the various marginal modes involved is described in Sect. 4. The numerically obtained results are described and discussed in Sect. 4. The paper ends with some concluding remarks, in Sect. 5.

\section{Compressible Navier-Stokes Equations}

The continuity, momentum, and energy equations are nondimensionalized using the streamwise length $\hat{x}_{0}$, outer velocity $\hat{u}_{0}$, density $\hat{\rho}_{0}$, and temperature $T_{0}$ as characteristic length, velocity, density, and temperature, respectively; time and the modified pressure $\left(=\right.$ pressure $\left.-\hat{\rho}_{0} R \hat{T}_{0}\right)$ are nondimensionalized with $\hat{x}_{0} / \hat{u}_{0}$ and $\hat{\rho}_{0} \hat{u}_{0}^{2}$, respectively. With the usual notation, the resulting equations are

$$
\begin{gathered}
\frac{\partial \rho}{\partial t}+\nabla \cdot(\rho \boldsymbol{v})=0, \quad 1+\mathcal{B}_{1} p=\rho T, \\
\frac{\partial \boldsymbol{v}}{\partial t}+(\boldsymbol{v} \cdot \boldsymbol{\nabla}) \boldsymbol{v}=-\boldsymbol{\nabla} p+\frac{1}{\operatorname{Re}}\left[\boldsymbol{\nabla} \cdot\left[M\left(\boldsymbol{v}+\boldsymbol{v}^{\top}\right)\right]-\frac{2}{3} \boldsymbol{\nabla}(M \boldsymbol{\nabla} \cdot \boldsymbol{v})\right], \\
\rho \frac{\mathrm{D} T}{\mathrm{D} t}=\boldsymbol{\nabla} \cdot\left[\frac{M}{\operatorname{Pr} R e} \nabla T\right]+\mathcal{B} \frac{\mathrm{D} p}{\mathrm{D} t}+ \\
\frac{\mathcal{B} M}{\operatorname{Re}}\left\{\boldsymbol{\nabla} \cdot\left[\boldsymbol{v} \cdot\left(\boldsymbol{\nabla} \boldsymbol{v}+\boldsymbol{\nabla} \boldsymbol{v}^{\top}\right)\right]-\boldsymbol{v} \cdot \boldsymbol{\nabla} \cdot\left(\boldsymbol{\nabla} \boldsymbol{v}+\boldsymbol{\nabla} \boldsymbol{v}^{\top}\right)\right\},
\end{gathered}
$$

in terms of the velocity $\boldsymbol{v}=(u, v)$, the density $\rho$, the modified pressure $p$, and the temperature $T$, where the Re and $M a$ are the Reynolds and Mach numbers based on the conditions at the outer flow, and $\operatorname{Pr}$ is the Prandtl number, which can be considered as constant $\operatorname{Pr}=0.72$ for air.

$$
\mathcal{B}=(\gamma-1) M a^{2} \text { and } \quad \mathcal{B}_{1}=\gamma M a^{2}
$$

are nondimensional measures of viscous dissipation and compressibility, respectively, where $\gamma=1.4$ for air. The function $M$, results from the dependence of viscosity on temperature, assumed to obey a Sutherland formula, which gives

$$
M(T)=\frac{T^{3 / 2}(1+s)}{T+s},
$$

with $s=\hat{s} / \hat{T}_{0}$ and $\hat{s}=110 \mathrm{~K}$. Thus, e.g., $s=0.37$ if $\hat{T}_{0}=300 \mathrm{~K}$. The computational domain and the boundary conditions depend on the various approximations made below. 


\section{Mode Interaction; Parametric Forcing}

In a boundary layer above a plate at zero incidence, with a local thickness

$$
\delta=\sqrt{x / R e} \ll 1,
$$

a self-similar, approximated steady state solution exists,

$$
\left(\rho_{S}, u_{S}, v_{S}, T_{S}, p_{S}\right)=(\rho(\zeta), u(\zeta), \delta V(\zeta), p, t(\zeta)
$$

where

$$
\zeta=y / \delta
$$

Replacing these into (1)-(3) and neglecting $O(1 / R)$-terms, where

$$
R=R e \delta \equiv \sqrt{x R e} \ll 1
$$

is the Reynolds number based on the local boundary layer thickness, we obtain the following ODE system

$$
\begin{aligned}
& -(\rho u)^{\prime}+2(\rho V)^{\prime}=0, \quad \rho T=1 \\
& \rho(-\zeta u+2 V) u^{\prime}=2\left[M(T) u^{\prime}\right]^{\prime} \\
& \rho(-\zeta u+2 V) T^{\prime}=2 \operatorname{Pr}^{-1}\left[M(T) T^{\prime}\right]^{\prime}+2 \mathcal{B} M(T)\left(u^{\prime}\right)^{2} .
\end{aligned}
$$

The boundary conditions are

$$
u=v=T^{\prime}=0 \quad \text { at } \zeta=0, \quad u_{S}=1, \quad T=T_{e} \quad \text { at } \zeta=\infty,
$$

where the outer flow temperature $T_{e}$ is predetermined, and we are assuming a thermally insulated wall, namely assuming that the steady state is reached after a transient in which thermal equilibrium between the solid and the air is reached.

The stability of this self-similar steady state is analyzed considering two kinds of modes. $\mathrm{K}$ modes are, in some sense, the natural modes of the boundary layer because they exhibit the same scaling (7)-(8) as the basic steady state. These modes are nearly marginal (namely, exhibit a zero growth rate), exhibit a power law growth along the streamwise coordinate [L00], and play a secondary role in the transition process: they can either enhance [R01] or delay [CB01] transition. TS modes, instead, exhibit a streamwise wavelength comparable to the boundary layer thickness, and are analyzed setting

$$
(\rho, u, v, T, p)=(\tilde{\rho}, \tilde{u}, \tilde{v}, \tilde{T}, \tilde{p})+A(r, \phi, \delta \psi, \theta, \pi) \mathrm{e}^{\mathrm{i}\left(\int_{0}^{x} \varphi \mathrm{d} x-\omega t\right) / \delta}+\text { c.c. }
$$

where c.c. stands for the complex conjugate, the complex wavenumber $\alpha=$ $\varphi^{\prime}$, and the complex amplitude $A$ is allowed to depend slowly on $x$ and $t$. Substituting these into (1)-(3), linearizing and retaining $O(1 / R)$-terms, we obtain 


$$
\begin{aligned}
& \mathrm{i}(\alpha \tilde{u}-\omega) r+(\tilde{\rho} \psi)^{\prime}+\mathrm{i} \alpha \tilde{\rho} \phi=0, \quad \mathcal{B}_{1} \pi=\tilde{T} r+\tilde{\rho} \theta, \\
& 3 \mathrm{i} \tilde{\rho}(\alpha \tilde{u}-\omega) \phi+3 \tilde{\rho} \tilde{u}^{\prime} \psi=-3 \mathrm{i} \alpha \pi+R^{-1}\left[3\left(\tilde{M} \phi^{\prime}\right)^{\prime}+\tilde{M}\left(\mathrm{i} \alpha \psi^{\prime}-4 \alpha^{2} \phi\right)\right. \\
& \left.\quad+3 \mathrm{i} \alpha \tilde{M}^{\prime} \tilde{T}^{\prime} \psi+3\left(\tilde{M}^{\prime} \tilde{u}^{\prime} \theta\right)^{\prime}\right], \\
& 3 \mathrm{i} \tilde{\rho}(\alpha \tilde{u}-\omega) \psi=-3 \pi^{\prime}+R^{-1}\left[4\left(\tilde{M} \psi^{\prime}\right)^{\prime}+\tilde{M}\left(\mathrm{i} \alpha \phi^{\prime}-3 \alpha^{2} \psi\right)\right. \\
& \left.\quad+\mathrm{i} \alpha \tilde{M}^{\prime}\left(-2 \tilde{T}^{\prime} \phi+3 \tilde{u}^{\prime} \theta\right)\right], \\
& \mathrm{i} \tilde{\rho}(\alpha \tilde{u}-\omega) \theta+\tilde{\rho} \tilde{T}^{\prime} \psi=\left(P r R^{-1}\right)\left[\left(\tilde{M} \theta^{\prime}\right)^{\prime}-\alpha^{2} \tilde{M} \theta+\left(\tilde{M}^{\prime} \tilde{T}^{\prime} \theta\right)^{\prime}\right] \\
& \quad+\mathrm{i} \mathcal{B}(\alpha \tilde{u}-\omega) \pi+\mathcal{B} R^{-1}\left[2 \tilde{M} \tilde{u}^{\prime}\left(\phi^{\prime}+\mathrm{i} \alpha \psi\right)+\tilde{M}^{\prime}\left(\tilde{u}^{\prime}\right)^{2} \theta\right],
\end{aligned}
$$

where $\tilde{M}$ denotes $M(\tilde{T})$. The appropriate boundary conditions are

$$
\phi=\psi=\theta=0 \quad \text { at } \zeta=0, \infty \text {. }
$$

Note that now (cf. (13)) we are assuming that the wall is isothermal, with the steady state temperature. This is because (a) the heat capacity and the thermal conductivity of the plate are both much larger than those of the air, and (b) the characteristic time of the nonsteady flow (essentially, the period of the Tollmien-Schlichting waves) is much smaller than the conductive time in the plate. Also note that we are retaining small $O(1 / R)$-terms, which account for viscous effects and are essential to trigger the instability that promotes TS waves; an asymptotic analysis as $R \rightarrow \infty$ of (15)-(19) leads to a triple-deck problem [S82], which requires to consider fractional powers of $R^{-1}$ and yields a poor approximation. Thus, the usual strategy is to retain $\mathrm{O}\left(R^{-1}\right)$-terms, as we do here, and solve numerically the resulting stiff problem. This can be done either discretizing the boundary value problem or using a shooting method combined with a continuous orthonormalization method [D83]; we have done the latter. In either case, the boundary conditions at $\zeta=\infty$ must be imposed at a (large but) finite distance, treated conveniently [K76] to avoid large errors due to wave reflection. Solving (15)-(19) yields marginal instability curves in the planes $\alpha$ vs. $R$ and $\omega$ vs. $R$ that are tongues like those shown in Fig. 1, where instability sets in when entering the tongues. Instability is convective and thus it can be seen as a spatial instability [Ch05], which develops with a fixed frequency $(\omega / \delta=\mathrm{real}=\mathrm{constant}$, see $(15))$. This means invoking (6)-(9) moving along a straight line passing through the origin in the $\omega$ vs. Re pane in Fig. 1.

Some remarks are now in order:

- As anticipated above, the real parts of $\alpha$ and $\omega$ (wavenumber and frequency) are much larger than the imaginary parts (spatial and temporal growth rates) inside the tongues in Fig. 1. This is illustrated in Fig. 2, 

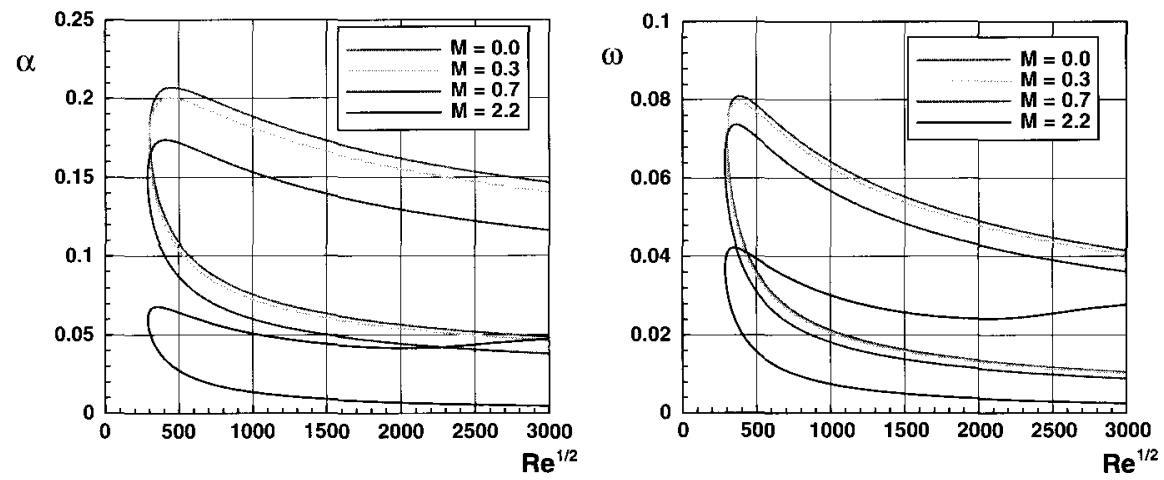

Fig. 1. Neutral stability curves
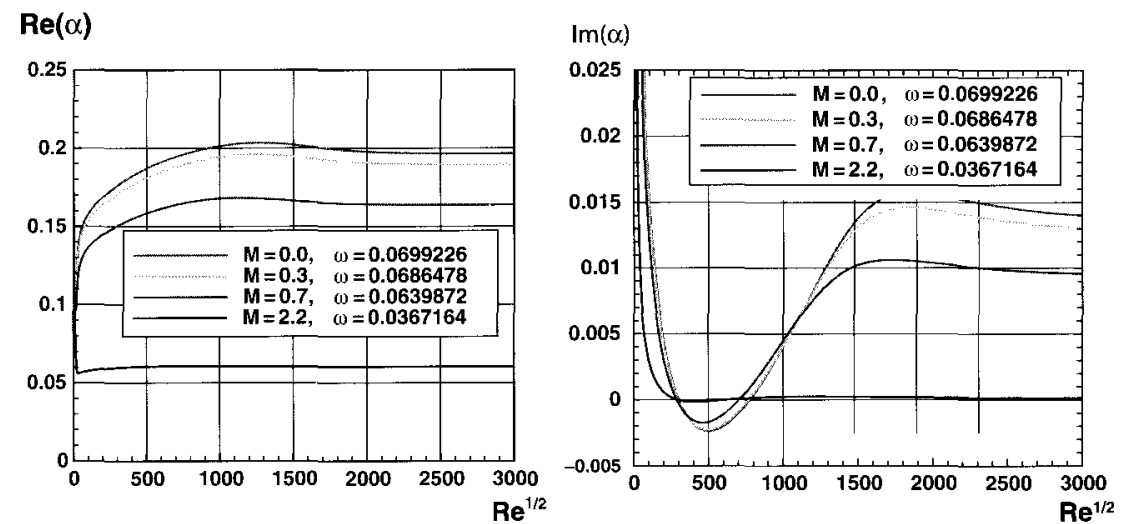

Fig. 2. Wavenumber and damping rate vs. $R=R e^{1 / 2}$ for fixed $\omega$

where the real and imaginary parts of $\alpha$ are plotted vs. $R$ for the indicated values of $\omega$ and $M$. This means, in particular, that the spatial evolution of these modes exhibit two well separated scales and can be described as slowly modulated wavetrains, of the form (14), with $\varphi$ and $\omega$ real and the complex amplitude $A$ satisfying a linear equation of the form

$$
A^{\prime}=\mu A,
$$

where the growth rate $\mu$ is such that $|\mu| \ll \varphi^{\prime} / \delta$.

- Parametric coupling between two TS modes, with frequencies and wavenumbers $\omega_{j}$ and $\kappa_{j}$ for $j=1$ and 2 , is promoted through (quadratic) nonlinear terms by a wavetrain in the boundary layer, with a frequency $\omega$ and wavenumber $\kappa$, such that

$$
\omega=\omega_{1}+\omega_{2}, \quad \kappa=\kappa_{1}+\kappa_{2}
$$


The complex amplitudes of both TS modes, $A_{1}$ and $A_{2}$ (which would obey equations of the type (20) in the absence of parametric coupling) evolve according to a system of coupled equations, of the form

$$
A_{1}^{\prime}=\mu_{1} A_{1}+\beta_{1} a \bar{A}_{2}, \quad A_{2}^{\prime}=\mu_{2} A_{2}+\beta_{2} a \bar{A}_{1},
$$

where $\mu_{1}$ and $\mu_{2}$ are the growth rates of the TS modes (both small), $a$ is the (small) amplitude of the wavetrain, and the complex coefficients $\beta_{1}$ and $\beta_{2}$ are of order one. Thus, effective parametric forcing requires that $|a| \sim\left|\mu_{j}\right|$, which according to our comment above requires that $a$ be small, of order 0.001 (see Fig. 2). This coupled evolution can be either more stable or more unstable than the original uncoupled evolution, depending on the coupling coefficients $\beta_{1}$ and $\beta_{2}$. Since the coupling coefficients depend on $x$, this cannot be elucidated analytically, but can be illustrated in the constant coefficient case, in which coupling stabilizes provided that the real part of $\beta_{1} \bar{\beta}_{2}$ be negative. It turns out that the wavetrain is stabilizing in all situations that have been checked.

- The parametric forcing described above is not the end of the mode interaction story. Once the two original TS modes and the wavetrain are present, nonlinearities force infinitely many new modes, with frequencies and wavenumbers $(j-k) \omega+\left(j_{1}-k_{1}\right) \omega_{1}+\left(j_{2}-k_{2}\right) \omega_{2}$ and $(j-k) \kappa+\left(j_{1}-k_{1}\right) \kappa_{1}+\left(j_{2}-k_{2}\right) \kappa_{2}$, for any natural numbers $j, k, j_{1}, k_{1}, j_{2}$, and $k_{2}$; the amplitudes are $\gamma a^{j} \bar{a}^{k} A_{1}^{j_{1}} \bar{A}_{1}^{k_{1}} A_{2}^{j_{2}} \bar{A}_{2}^{k_{2}}$, where $a, A_{1}$, and $A_{2}$ are the (small) complex amplitudes of the wavetrain and the TS modes, and the coefficient $\gamma$ is $O(1)$ if the excited mode is not a nearly marginal mode, but can be large otherwise. The latter case is that in which the mode interaction process is effective. For instance, in the parametric interaction case above, $j=1, j_{1}=-1, k=k_{1}=j_{2}=k_{2}=0$ and the excited mode is the second TS mode, which is nearly marginal. Some additional, not so strong resonances can also appear, see Fig. 5 in Sect. 4 . But there is an additional resonance that is always present and is associated with a mean flow. If

$$
\left|A_{1}\right| \sim\left|A_{2}\right| \ll a \ll 1,
$$

the mean flow is produced mainly by the wavetrain, and exhibits an amplitude that is of the order of $R a^{2}=a^{2}$, which can affect the stability of the TS waves. It turns out that the effect of the mean flow is taken into account replacing (22) by

$$
A_{1}^{\prime}=\left(\mu_{1}+\beta_{3} R a^{2}\right) A_{1}+\beta_{1} a \bar{A}_{2}, \quad A_{2}^{\prime}=\left(\mu_{2}+\beta_{4} R a^{2}\right) A_{2}+\beta_{2} a \bar{A}_{1},
$$

with the complex coefficients $\beta_{3}$ and $\beta_{4}$ of order one. The coefficients in this equation can be obtained via weakly nonlinear analysis, which is omitted here. Instead, we use (24) to guess the order of magnitude of the various amplitudes for these mode interaction be effective, namely

$$
\left|\mu_{1}\right| \sim\left|\mu_{2}\right| \sim|a| \sim R a^{2} \ll 1 .
$$

This will be used in the DNS analysis that is considered next. 


\section{Direct Numerical Simulation}

In a flat plate at zero incidence boundary layer, (1)-(3) should be integrated in a domain close to the plate and extending streamwise to a position beyond transition, which occurs at a fairly high Reynolds number $\left(\sim 5 \times 10^{6}\right)$. Since the smallest scale associated with the viscous sublayer must be described, this is quite costly numerically. Thus, we take a computational domain in the streamwise direction covering only a portion of the boundary layer and impose the steady profile at the entrance. Namely, the computational domain is

$$
x_{0}<x<x_{0}+L, \quad 0<y<y_{0},
$$

with $L \ll x_{0}$ but somewhat large as to include several wavelengths of the relevant waves, and $y_{0}$ somewhat large compared to the boundary layer thickness. In this region, (1)-(3) apply, with the Reynolds number $R$ based on the distance from the leading edge to the entrance of the computational domain. For convenience, we first calculate the steady state solution, $\left(\boldsymbol{v}_{s}, \rho_{s}, T_{s}\right)$, with boundary conditions

$$
\begin{aligned}
& \boldsymbol{v}_{s}=\tilde{\boldsymbol{v}}(y), \quad \rho_{s}=\tilde{\rho}(y), \quad T_{s}=\tilde{T}(y) \quad \text { at } x=x_{0}, \\
& u=1, \quad \partial v / \partial y=\partial \rho / \partial y=\partial T / \partial y=0 \quad \text { at } y=y_{0}, \\
& \boldsymbol{v}=0, \quad \partial T / \partial y=0 \quad \text { at } y=0 \\
& \partial \boldsymbol{v} / \partial x=0, \quad \partial T / \partial x=0 \quad \text { at } x=x_{0}+L
\end{aligned}
$$

where the plate is assumed to be thermically insulated, and $(\tilde{\boldsymbol{v}}, \tilde{\rho}, \tilde{T})$ is the Blasius self-similar steady state solution at $x=x_{0}$, given by ( 7 ).

The boundary conditions for the nonstationary problem are assumed to be such that:

- The temperature of the plate (and the air just above the plate) is assumed to remain at its steady state value. This is because the characteristic time in the air (associated with TS oscillations) is much shorter than the heat conduction time in the solid.

- The solution should match with the uniform flow outside the boundary layer. When imposing boundary conditions at a finite distance from the plate, spurious reflection of both acoustic and hydrodynamic waves must be avoided.

- In order to generate a TS wave in the boundary layer, a vibrating membrane can be used with a vibrating frequency equal to that of the TS wave; see (33) and (35) below. The size of the membrane is not essential (because the spatially parabolic character of the boundary layer). A size similar to the wavelength of the TS wave is nevertheless convenient to facilitate generation.

- In order to generate a wavetrain in the air, a wavetrain-like boundary condition for the vertical velocity is imposed in the plate; see (33)-(36). This can be achieved in practice using a periodic array of oscillators, one at each period of the wavetrain, with a frequency $\omega$ and appropriate phase shifts. 
- The boundary conditions at the exit are unessential (again, because of the parabolic character of the boundary layer), but a buffer near the exit (where the solution depends on the selected boundary conditions) must be excluded from post processing.

With these ideas in mind, the boundary conditions for the nonstationary problem are (cf. (27)-(30))

$$
\begin{aligned}
& \boldsymbol{v}_{s}=\tilde{\boldsymbol{v}}(y), \quad \rho_{s}=\tilde{\rho}(y), \quad T_{s}=\tilde{T}(y) \quad \text { at } x=x_{0}, \\
& u=1, \quad \partial v / \partial y=\partial \rho / \partial y=\partial T / \partial y=0 \quad \text { at } y=y_{0}, \\
& u=0, \quad v=v_{0}(x, t) T=T_{s}(x, 0) \quad \text { at } y=0, \\
& \partial \boldsymbol{v} / \partial x=\mathbf{0}, \quad \partial T / \partial x=0 \quad \text { at } x=x_{0}+L,
\end{aligned}
$$

where

$$
\begin{aligned}
& v_{0}(x, t)=\varepsilon \sin \kappa_{1}\left(x-x_{1}\right) \sin \omega_{1} t \quad \text { if }\left|x-x_{1}\right|<\pi / \kappa_{1}, \\
& v_{0}(x, t)=a \sin (\kappa x-\omega t) \quad \text { if } x_{2}<x<x_{0}+L \\
& v_{0}(x, t)=0 \quad \text { if either } x_{0}<x \leq x_{1}-\pi / \kappa_{1}, \text { or } x_{1}+\pi / \kappa_{1} \leq x<x_{0}+L .
\end{aligned}
$$

Now, the numerical tool must be sufficiently precise as to give a precise description of the TS modes involved, which exhibit quite small amplitude and growth rates; in particular, numerical viscosity must be quite small. Also, in order to isolate the contribution of the various modes in the complete flow field provided by the numerical tool, a temporal fast Fourier transform tool is used that gives the components of the flow at various frequencies.

Now, we are in a position to simulate the mode interaction process explained in Sect. 3 and illustrated in Fig. 3.

To this end, we perform the following simulations at $M a=0.3, R e=$ $1.96 \times 10^{7}$ (which gives $R e \simeq 4,430$ using $\left.(9)\right), \Omega_{1}=\Omega_{\mathrm{TSu}}=90, \kappa_{1}=\kappa_{T S u}=$ 410 (frequency and wavenumber of an unstable TS mode, with growth rate $d=29), \Omega_{2}=\Omega_{\mathrm{TSs}}=150, \kappa_{2}=\kappa_{\mathrm{TSs}}=440$ (stable TS mode, with $d=-42$ ), $\varepsilon=2 \times 10^{-3}$, and various values of $a$.

If $a=0$, then only the unstable TS mode is forced (Fig. 3 top). In order to check the numerical approximation, the horizontal velocity profile of the TS mode is compared in Fig. 4 top with its counterpart obtained from the linear approximation. (15)-(19). If $\varepsilon=0, a=0.001, \Omega=240$, and $\kappa=850$, then we only generate a wavetrain (Fig. 3 middle). The numerical approximation is now checked in Fig. 4 bottom, where the linear approximation of the wavetrain is calculated using (15)-(19), except the boundary condition for the vertical velocity at $\zeta=0$, which is replaced by $\psi=a$.

If both $\varepsilon \neq 0$ and $a \neq 0$, then since the frequencies and wavenumbers satisfy the resonance relations (21), the stable TS is also forced parametrically 


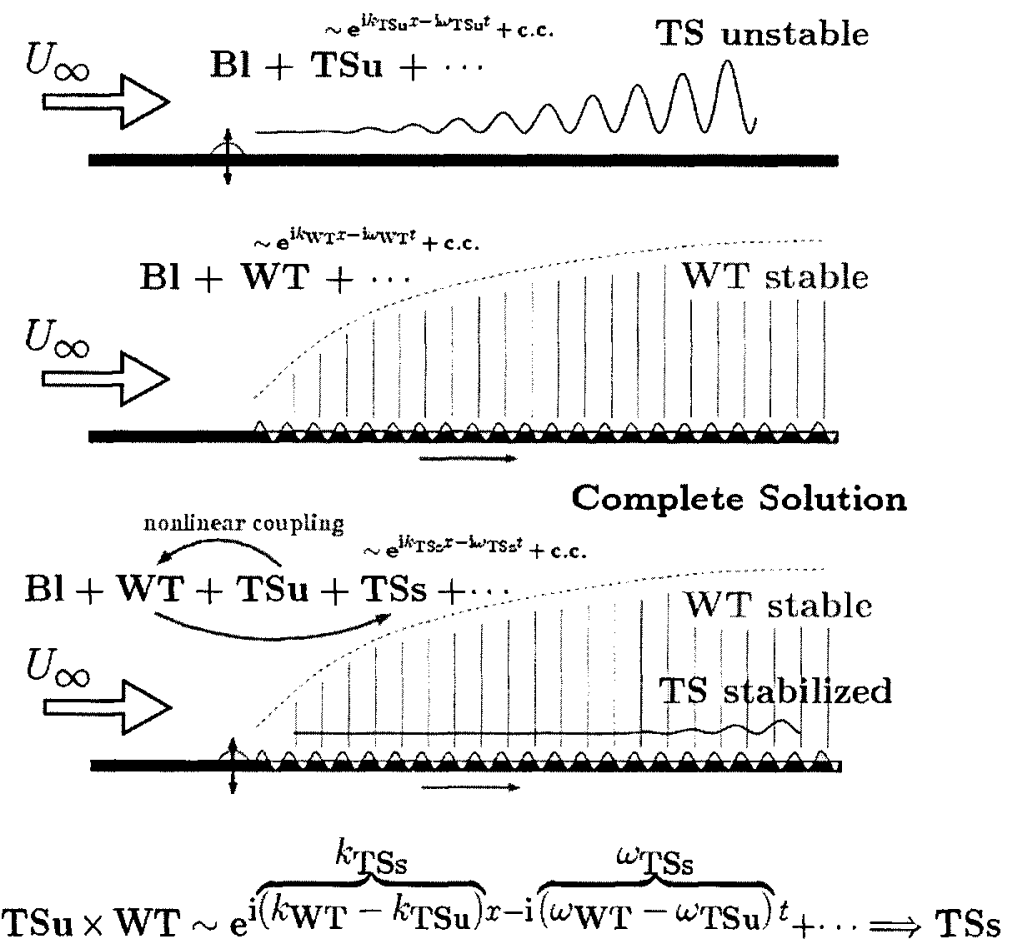

Fig. 3. The mode interaction process. Top: unstable TS wave, TSu, generated with an actuator. Middle: generation of a wavetrain, WT, at the bottom of the plate (red). Bottom: the WT induces a nonlinear coupling between a stable TS (TSs) and unstable TS, reducing spatial growth
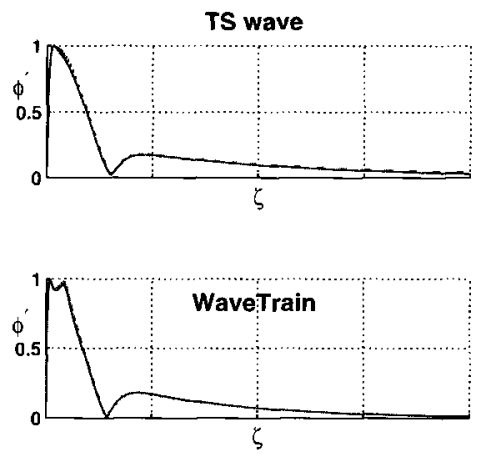

Fig. 4. Comparison of the horizontal velocity profiles provided by DNS and linear stability for the unstable TS mode with $\Omega=90$ (top) and the wavetrain obtained with $\Omega=240$ and $\kappa=850$ (bottom) 

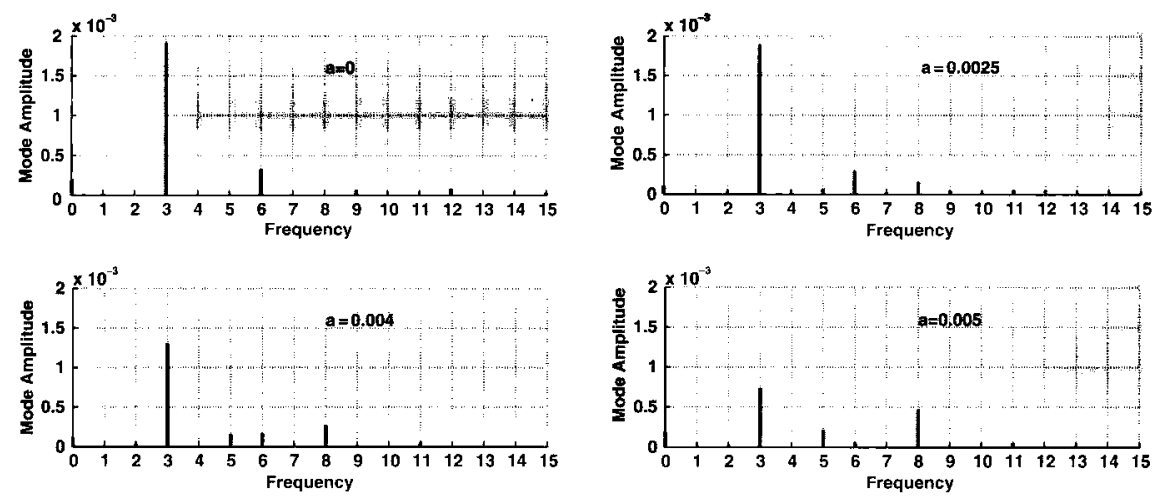

Fig. 5. Temporal fast Fourier transform (FFT), with frequency $=\Omega / 30$ in abscissa. Thus the unstable and stable TSs, and the wavetrain correspond to frequencies 3 , 5 , and 8 , respectively
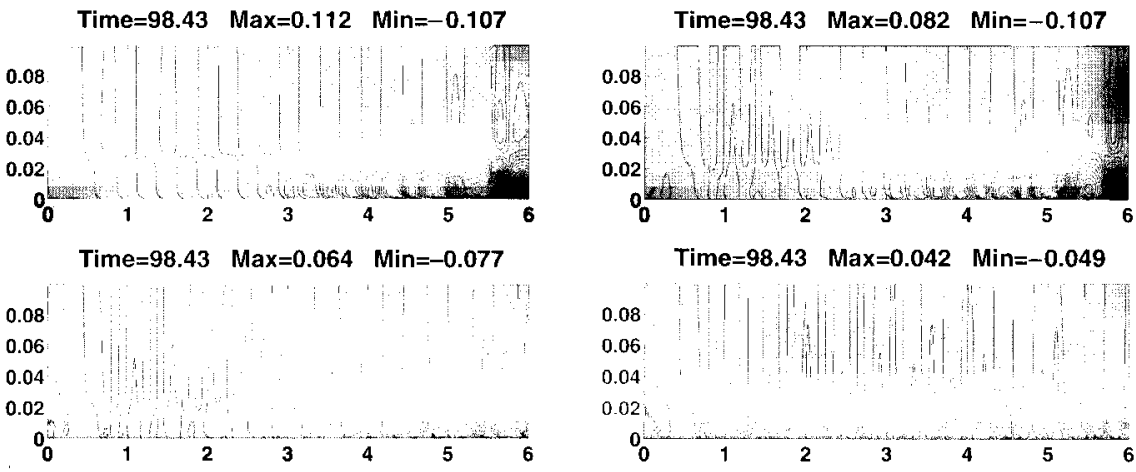

Fig. 6. Streamwise perturbation velocity contours for the WT wave amplitudes corresponding to the dots marked in Fig. 5 ( $a=0,0.0025,0.004$, and 0.005 from left to right and top to bottom)

and its spatial evolution is coupled with that of the unstable TS, as explained in Sect. 3. This is illustrated in the FFT plots in Fig. 5, where it is seen that if $a=0$ the unstable TS mode (with $\Omega=90$ and its harmonics appear while for increasing values of $a$, both the wavetrain (with $\Omega=240$ ) and the stable TS mode (with $\Omega=150$ ) are also present with increasing amplitude, while the amplitude of the unstable TS mode decreases, meaning that the latter is stabilized. In particular, at $a=0.005$ the unstable TS mode has been divided by three. Note that the mean flow corresponds to $\Omega=0$ and remains small for all considered values of $a$. This is because since $R e=4,430$, $a^{2} R e \ll 1$ for the considered values of $a$, and that term accounting for the mean flow in (24) is much smaller than that term accounting for parametric forcing. The mean flow seems to give additional stabilization at $a=0.005$, but this should be checked. This stabilization process is further illustrated in the snapshots plotted in Fig. 6, where it is clearly appreciated that the strength 
of the unstable TS mode at $a=0$ is strongly reduced by the presence of the wavetrain. Thus, this mode interaction process is quite effective.

\section{Concluding Remarks}

We have applied a mode interaction process to stabilize TS waves in a compressible, 2D boundary layer attached to a flat plate at zero incidence. In order to stabilize a given unstable TS wave, a wavetrain is created in the boundary layer that couples parametrically the spatial evolution of the unstable TS wave with the evolution of a stable TS wave, in such a way that the stable takes energy from the unstable and stabilizes it. The process has been explained qualitatively in Sect.3, where the required order of magnitude of the amplitude, frequency, and wavenumber of the stabilizing wavetrain was anticipated. In addition, we also anticipated that the mean flow produced by the wavetrain can also play a role. All these has been confirmed in Sect. 4, where a battery of numerical simulation was performed. Additional DNS simulations, not presented here, show that the process is quite robust in the sense that it is fairly insensitive to both the frequency and wavenumber of the wavetrain. This must be because of the stalibilizing effect of the wavetrain, which does not depend on any resonance relation and seems to also contribute to stabilizing the system. But the analysis of this is beyond the scope of this paper.

\section{References}

[Ch05] Chomaz, J.M.: Global instabilities in spatially developing flows: nonnormality and nonlinearity, Annu. Rev. Fluid Mech., 37, 357-392 (2005)

[CB01] Cossu, C. and Brant, L.: Stabilization of Tollmien-Schlichting waves by finite amplitude optimal streaks in a Blasius boundary layer. Phys. Fluids, 14, 016318-1-17 L.57-L.60(2002)

[D83] Davey, A.: An authomatic orthonormalization method for solving stiff boundary-value problems, J. Compt. Phys., 51, 343-356 (1983)

[J98] Joslin, R.D.: Aircraft laminar flow control. Annu. Rev. Fluid Mech., 30, $1-29$ (1998)

[K94] Kachanov, Y.S.: Physical mechanisms of laminar boundary layer transition. Annu. Rev. Fluid Mech., 26, 411-482 (1994)

[K76] Keller, H.: Numerical Solutions of Two Point Boundary Value Problems, Vol.24 of CBMS-NSF Regional Conferences in Applied Mathematics, SIAM, 1976.

[LG06] Li, Y., Gaster, M.: Active control of boundary layer instabilities. J. Fluid Mech., 550, 185-205 (2006)

[LMV01] Lapuerta, V., Mancebo, F.J. and Vega, J.M.: Control of Rayleigh-Taylor instability by vertical vibration in large aspect ratio containers. Physical Review E, 64, 016318-1-17 (2001) 
[L00] Luchini, P.: Reynolds number independent instability of the boundary layer over a flat plate: optimal perturbations. J. Fluid Mech., 404, 289 309 (2000)

[R01] Reshotko, E.: Transient growth: A factor in bypass transition. Phys. Fluids, 13, 1067-1075 (2001)

[SG00] Schlichting, H., Gersten, K.: Boundary layer theory. Springer, Berlin (2000)

[S82] Smith, F.T.: On the high Reynolds number theory of laminar flows. IMA J. Appl. Math., 28, 207-281 (1982)

[WAA01] Walther, S., Airiau, C., Bottaro, A.: Optimal control of TollmienSchlichting waves in a developing boundary layer. Phys. Fluids, 13, 20872096 (2001)

[ZLB04] Zuccher, S, Luchini, P. Bottaro, A.: Algebraic growth in a Blasius boundary layer: optimal and robust control by mean of suction in the nonlinear regime. J. Fluid Mech., 513, 135-160 (2004) 